\title{
Status and prospects for the PADME experiment at LNF
}

\author{
Paola Gianotti ${ }^{1, \star}$

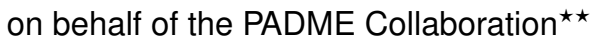 \\ ${ }^{1}$ Laboratori Nazionali di Frascati dell'INFN, via E. Fermi 40, I-00044 Frascati, Italy
}

\begin{abstract}
The PADME collaboration aims to search for signals of a light dark photon $A^{\prime}$ using the beam of the LNF LINAC. The experiment, approved by INFN at the end of 2015 , foresees to detect $A^{\prime}$, produced in the annihilation of positrons on a thin fixed target, by searching for missing mass signals. The detector construction is ongoing and should be completed within the end of 2017 in order to allow the collection of about $10^{13}$ positrons on target which are necessary to get a $10^{-3}$ sensitivity on the mixing parameter $\varepsilon$ up to a dark photon mass of $23.7 \mathrm{MeV} / c^{2}$.
\end{abstract}

\section{Introduction}

Direct observations of dark matter signals are necessary to shed light on the cosmological and astrophysical evidences, in order to understand the nature of such unknown matter that at present has manifested itself only via gravitational interactions.

One of the present theoretical hypothesis foresees that the new dark particles are not directly connected with the Standard Model (SM) gauge fields, but only through mediators or "portals", linking our world with the new "secluded" sector [1]. A simple model that tries to explain dark matter, just adds an additional $U(1)$ symmetry, with its corresponding vector boson $A^{\prime}$. All SM particles are neutral under this symmetry, and the new field couples to the charged particles of the SM with an effective charge $\varepsilon q$, where $q$ is the electromagnetic charge, induced by the kinetic mixing of the dark photon with the SM photon. If this dark photon $A^{\prime}$ would have a mass in the range from 1 to $100 \mathrm{MeV} / \mathrm{c}^{2}$ and a coupling $\varepsilon \sim 10^{-3}$, it could also explain the discrepancy between theory and observation for the muon anomalous magnetic moment [2]. This possibility has been recently disproved under the assumption that the $A^{\prime}$ decays to SM particles only (for a recent review see [3]), but if the $A^{\prime}$ decays to dark sector particles, almost all of the available experimental constraints can be evaded and the dark photon is still a valuable explanation for the muon $(g-2)$ anomaly.

To test this hypothesis, PADME (Positron Annihilation into Dark Matter Experiment) will search for invisible decays of the $A^{\prime}$ at the LNF LINAC. The experiment is designed to detect dark photons

\footnotetext{
^e-mail: paola.gianotti@lnf.infn.it

${ }^{\star}$ G. Chiodini (INFN Lecce), A.P. Caricato, M. De Feudis, M. Martino, G. Maruccio, A. Monteduro, S. Spagnolo (INFN Lecce and Dip. Matematica e Fisica Università del Salento), R. Bedogni, B. Buonomo, F. Bossi, P. Gianotti, R. De Sangro, G. Finocchiaro, L.G. Foggetta, A. Ghigo, M. Palutan, G. Piperno, B. Sciascia, T. Spadaro (INFN Laboratori Nazionali di Frascati), G.V. Georgiev, V. Kozhuharov (University of Sofia and INFN Laboratori Nazionali di Frascati), U. Dosselli (INFN Padova), F. Ferrarotto, E. Leonardi, P. Valente (INFN Roma1), S. Fiore (INFN Roma1 and ENEA), G.C.Organtini, M.Raggi (INFN Roma1 and Dip. di Fisica Università "Sapienza" di Roma )
} 
produced in positron on fixed target annihilation $e^{+} e^{-} \rightarrow \gamma A^{\prime}$ by measuring the final state missing mass.

The collaboration aims to complete the design and construction of the detector by the end of 2017 and to collect $\sim 10^{13}$ positrons of $550 \mathrm{MeV}$ energy on target by the end of 2018 . This will allow to reach a sensitivity $\varepsilon \sim 10^{-3}$ on the coupling, for dark photon masses up to $23.7 \mathrm{MeV} / c^{2}$.

In the following sections more details on the detector design and on the experiment schedule are given.

\section{The PADME detector}

The goal of the PADME experiment is to search for dark photons produced in the annihilation process of the positron beam of the DAФNE LINAC with a thin carbon target and then identified using the missing mass technique [4]. The ordinary recoil photon is detected by a finely segmented electromagnetic calorimeter that also measures the particle four-momentum with high precision. This approach allows to identify the dark photon in case it is a stable particle, or if it decays to invisible particles, and also in case of visible decays, by disregarding the decay products of the dark photon. With the present foreseen positron energy of the LNF LINAC of $550 \mathrm{MeV}$, masses up to $23.7 \mathrm{MeV} / c^{2}$ could be explored. This region is currently unconstrained by direct measurement, and PADME has the unique opportunity to be the first dedicated experiment to search directly for the dark photon invisible decays in a model-independent way.

The basic elements of the PADME experiments are:

- a high intensity and low divergence positron beam, impinging on a thin, active target, capable of monitoring the beam spot;

- a vacuum chamber to avoid spurious positron interactions;

- a magnet to deflect the beam of positrons emerging from the target, with the additional task of measuring the momentum of the interacting positrons, thus improving the rejection of the Bremsstrahlung background;

- a finely-segmented, high-resolution e.m. calorimeter, to measure the momentum of the single SM photons.

A schematic view of the PADME layout can be seen in fig. 1.

The processes that will mainly take place in the beam-target interaction are Bremsstrahlung and $e^{+} e^{-} \rightarrow \gamma \gamma(\gamma)$. In order to cut out these background events, two extra components are crucial: a fast Small Angle Calorimeter (SAC) and a charged particle veto system. Fig. 2 shows the results of Montecarlo simulations performed to check background rejection capability requiring only one cluster in the main calorimeter (with energy in a range optimized depending on $m_{A^{\prime}}$ ), no hits in the vetoes, and no photons with energy $>50 \mathrm{MeV}$ in the SAC.

\subsection{The positron beam}

The PADME positron beam will be extracted from the warm LNF LINAC of the DAФNE complex using the BTF (Beam Test Facility) line [5]. The typical size of the beam spot at the BTF is below $1 \mathrm{~cm}^{2}$ RMS, while the energy spread is at the level of $1 \%$. At present, this line is tuned to provides pulses at $510 \mathrm{MeV}$ with a maximum length of $40 \mathrm{~ns}$ and a repetition rate up to $50 \mathrm{~Hz}$. This is far from the requirements of the experiment where the interaction rate is ultimately dictated by the pileup probability in the downstream calorimeters. The ideal PADME beam would be continuous or quasi-continuous, with an average time distance between two positrons larger than the characteristic 


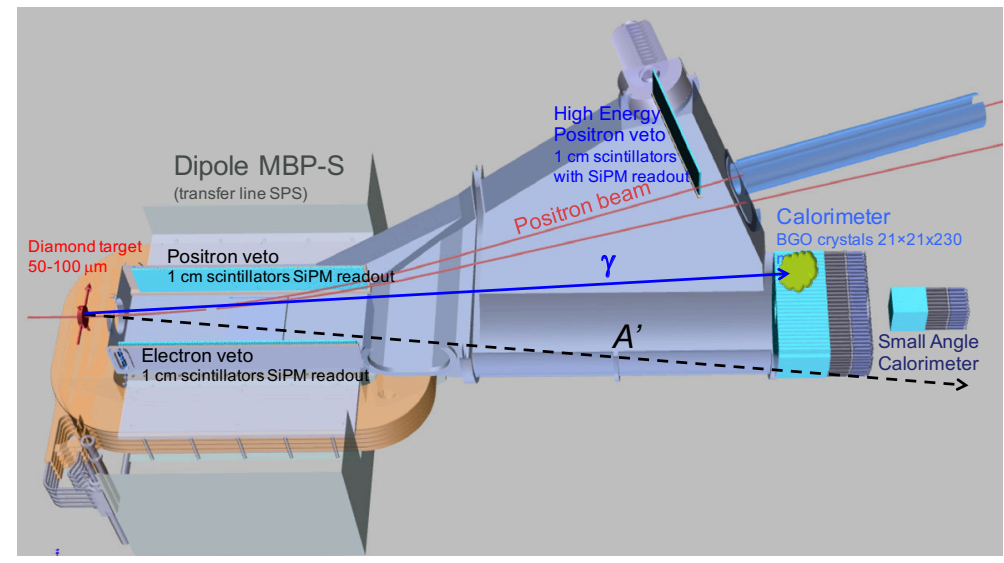

Figure 1: PADME detector layout. From left to right: the active target, the positron/electron vetoes inside the magnetic dipole, the high energy $e^{+}$veto near the non-interacting beam exit, the e.m. calorimeters. The distance from the target to the first calorimeter is roughly $3 \mathrm{~m}$.

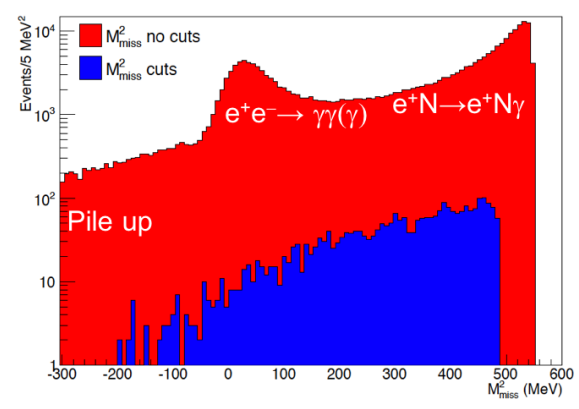

Figure 2: Montecarlo simulated squared missing mass for background events. In red with no selection cuts, in blue after all cuts are applied (see text for more details).

response time of the calorimeters. This can be achieved by reducing the beam intensity, but at the cost of increasing the running time, or by streaching the bunch. In practice, to perform the PADME experiment at the DAФNE LINAC, the beam intensity and width have to be tuned exploiting the flexibility of the BTF beam transport system. During 2016, a new LINAC pulsing system has been installed to increase pulse duration. A dedicated machine development run has been performed and pulses of electrons of $250 \mathrm{~ns}$ have been produced. Further adjustments are foreseen to reduce the intensity variations and to keep the beam energy spread below $2 \%$.

\subsection{The PADME magnet}

The positron beam will interact with a thin $(\sim 50-100 \mu \mathrm{m})$ carbon target passing through almost undisturbed. To drive away from the calorimeters acceptance these primary positrons, a magnetic field is necessary. Taking into account the PADME layout, this turned out in a dipole with a gap of $\pm 10 \mathrm{~cm}$ over a length of $1 \mathrm{~m}$, providing a field of about $0.5 \mathrm{~T}$. 
The magnet, besides sweeping the primary beam, will play an important role in the background suppression. Scintillator strips equipping the sides of the dipole will detect and will measure the momentum of the positrons that have lost a significant fraction of their energy by Bremsstrahlung in the target.

A magnet with the requested characteristics has been found at CERN within the spare dipoles of the SPS transport line (MBP-S), and it has been shipped to LNF at the end of 2015. During 2016, the dipole vertical gap has been increased to $230 \mathrm{~mm}$, to better suite the experiment needs, and the field map has been measured in different conditions (see fig. 3).
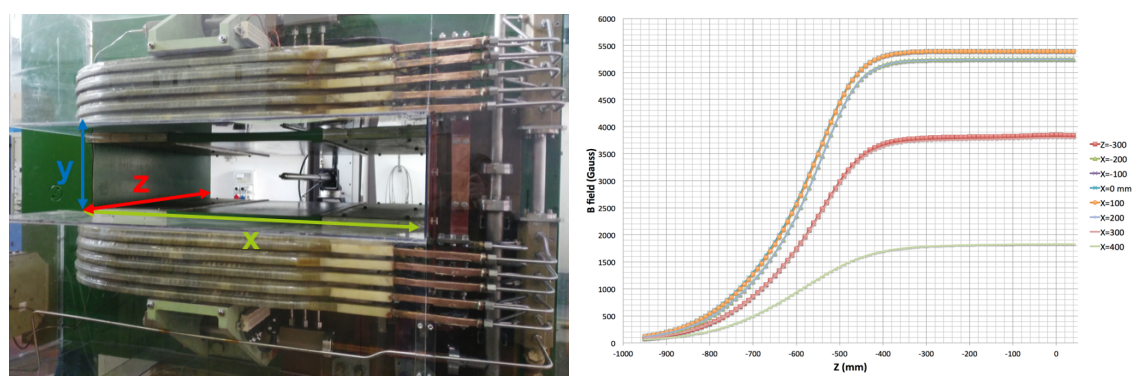

Figure 3: left the PADME magnet. right Longitudinal scan of magnetic field at different transverse positions.

\subsection{The diamond target}

For dark photon production and beam monitoring, a low- $Z$ active thin target is necessary. The low$\mathrm{Z}$ requirement, necessary to keep Bremsstrahlung at an acceptable level, combined with the need to monitor both the beam spot and intensity, has oriented the choice toward polycrystalline diamond sensors having on both sides orthogonal graphite strips for the readout. The advantage of this technique, developed by INFN Lecce [6], with respect to traditional metallic electrodes, lies in the fact that the target is entirely made of a homogeneous light material. The target thickness will be between 50 and $100 \mu \mathrm{m}$, to minimize the photon reinteraction, and the area will be about $2 \times 2 \mathrm{~cm}^{2}$, to match the size of the beam spot.

A first prototype of this target, $50 \mu \mathrm{m}$ thick and with a strip pitch of $1 \mathrm{~mm}$, has been built and tested at the BTF using positrons and electrons of $450 \mathrm{MeV}$ (see fig. 4). The results of the test beam show a very good spatial resolution $(0.2 \mathrm{~mm}$ RMS $)$ for a beam intensity of $10^{3}$ particles/bunch.

To further improve the beam monitor capability of the experiment, it is under development a system based on two planes of silicon pixel detectors placed up and down stream the diamond target. Each plane will consist of two MIMOSA 28 Ultimate chips, developed for the upgrade of the STAR vertex detector [7]. These devices integrate a Monolithic Active Pixel Sensor (MAPS) with a fast binary readout. Each sensor consists of a matrix of $928 \times 960$ pixels of $20.7 \mu \mathrm{m}$ with a thickness of 50 $\mu \mathrm{m}$. For the STAR experiment the chips, that dissipate $\sim 150 \mathrm{~mW} / \mathrm{cm}^{2}$, operate in air without cooling. For PADME, the detectors should be placed in vacuum and a modified PCB is under development at the LNF electronics workshop. First prototypes of this new monitoring system will be tested during 2017. 


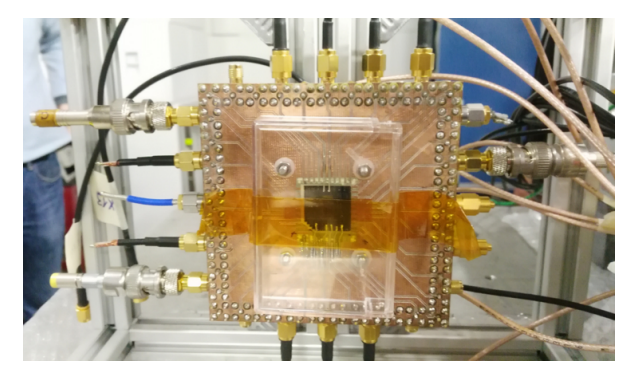

Figure 4: The first prototype of the PADME active target.

\subsection{The electromagnetic calorimeters}

The key point of the PADME experiment is to detect with high efficiency the energy, direction and timing of the single photons emitted from the target.

Therefore, e.m. calorimeters are necessary. The requirements are:

- high acceptance for the SM photons;

- energy resolution: $1-2 \% / \sqrt{E} \mathrm{GeV}$;

- time resolution $\sim 0.5 \mathrm{~ns}$;

- angular resolution $\sim 1 \div 2 \mathrm{mrad}$;

To avoid pile-up due to the high number of primary positrons, the main calorimeter (ECAL) will have a central hole to cut out most of the Bremsstrahlung photons produced in the interaction with the target. In the shadow of this hole, a second, small size and faster calorimeter (SAC), will be placed.

In the present design, the ECAL is a tight matrix of 616 crystals $\left(21 \times 21 \times 230 \mathrm{~mm}^{3}\right)$ arranged in a cylinder of external radius $30 \mathrm{~cm}$, with a central squared hole of $10 \mathrm{~cm}$ (see fig.5). The acceptance of the ECAL, for the recoil photons is $66 \%$, for a $550 \mathrm{MeV}$ positron beam.

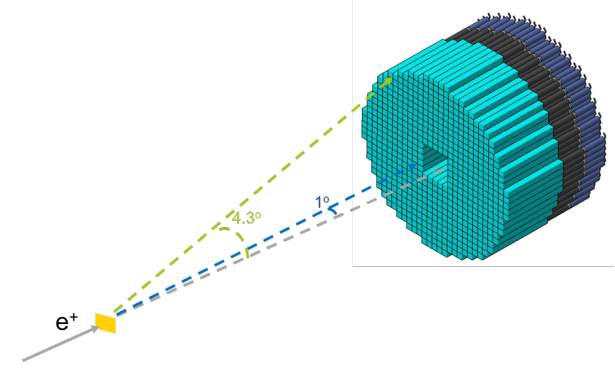

Figure 5: Layout of the PADME main e.m. calorimeter (ECAL). It consists of a tight matrix of 616 BGO crystals.

The choice of the scintillating material for the ECAL has been driven by different aspects: high density, small radiation length and Moliére radius (due to the calorimeter small dimensions), high light output (due to the low energy range), cost. Excluding the cost requirement, both LYSO and BGO would have been suitable, with a slight preference for the LYSO since it has a shorter decay 
time that would have mitigated the effect of event pileup caused by the beam structure. Nevertheless, the BGO crystals that were used by the L3 experiment at LEP [8], that are property of INFN, were available at the only cost of cutting them at the proper size. Therefore, after having checked that these crystals could be reconditioned and re-shaped, they have been adopted.

For the light collection, early tests, aimed at evaluating the best readout technology, showed that avalanche photodiodes (APDs), even with a relatively large area of $10 \times 10 \mathrm{~mm}^{2}$, have a gain that is insufficient to perform a high resolution energy measurement in the PADME energy rage (from a few to a few hundred MeV). The readout system will therefore be based on $19 \mathrm{~mm}$ diameter photomultipliers. On July 2016, XP1912 HZC Photonics tubes [9] have been tested at the BTF, on a calorimeter prototype made of $25 \mathrm{BGO}$ crystals obtained by machining the L3 recovered ones. The 25 channels of the prototype were fed into a CAEN V1742 high-speed digitizer [10], based on the DRS4 chip, set to a sampling speed of $1 \mathrm{GS} / \mathrm{s}$ (1 ns/sample).

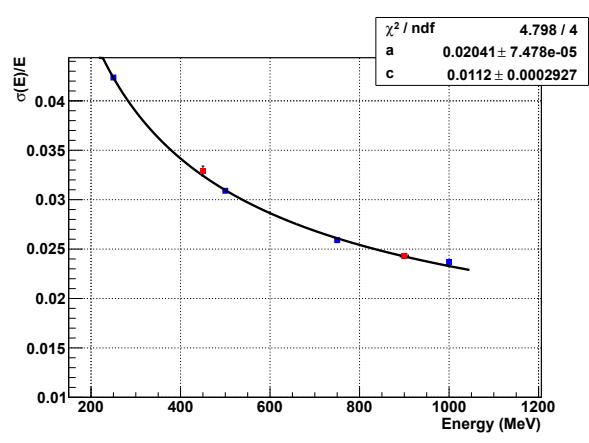

Figure 6: Energy resolution measured with a prototype of the PADME ECAL at the LNF BTF. Red and blue points refer to two different beam energies of 450 and $250 \mathrm{MeV}$, respectively. The line is the best fit curve whose parameters are quoted in the inset.

The test beam has demonstrated that the technical solutions chosen for this detector meet all the requirements. Fig. 6 shows the energy resolution obtained as a function of the photon energy. A complete overview of the test beam results can be found in [11].

Placed $50 \mathrm{~cm}$ downstream the ECAL, the SAC will be an array of fast detectors able to veto photons at small angles. $\mathrm{BaF}_{2}$ or lead glass are the crystal material candidates. For the readout, fast photomultipliers will be used. A test beam to select the final components will be performed at the LNF BTF in summer 2017.

\subsection{Charged particle vetoes}

The detection of the positrons that have lost energy in the interaction with the target, would significantly reduce the Bremsstrahlung background. Depending on the energy of the photon radiated, the corresponding positron can be inside the magnet acceptance or close to the initial beam trajectory. This is why it is planned to place on the side walls of the dipole, and close to the positron beam exit, 3 groups of scintillator strips with SIPM readout (see fig.1). The total number of strips $(10 \times 10 \times 200$ $\mathrm{mm}^{3}$ ) will be 250 (100 for the low energy positrons, 100 for the low energy electrons, 50 for the high energy positrons) and from the hit position a \% accuracy on the particle momentum is expected, provided a time resolution of about 300 ps. Tests with the BTF beams of the veto detectors prototype are foreseen in 2017. 


\section{Experiment time line}

After the approval at the end of 2015, an international collaboration has formed to perform the PADME experiment. At present it consists of about 30 people from 6 different institutions: INFN Lecce, Salento University, INFN LNF, University and INFN Roma, University of Sofia, MTA Atomki from Hungary. Contacts are ongoing with Cornell in the framework of the MAECI project PGR-226 and there are plans of cooperation to exchange expertise and knowhow. Cornell aims at creating an extracted positron beam line of $5.3 \mathrm{GeV}$ on which, if realized, an upgraded version of the PADME experiment could be installed.

The design of the detector elements has been optimized and frozen in almost all aspects, and the procurement of the main components has already started in 2016.

All the crystals, necessary for the ECAL, are now ready to be machined and coupled to the corresponding photomultipliers.

A new $100 \mu \mathrm{m}$ thick diamond target has been produced in Lecce and is currently under test with ${ }^{241}$ Am radioactive source. The design of the vacuum vessel section and the actuators for the target and beam diagnostics upstream of the magnet are now being finalized.

The design of the main vacuum vessel is presently being validated by an extensive simulation campaign, and the executive design will be frozen by the beginning of the 2017 , so that the construction can start.

The 15 tons magnet is ready to be moved to the BTF hall as soon as the experiment setup will be ready for the final installation.

The materials for the positron and electron scintillating veto detectors have been procured, and the construction will start in early 2017.

The requirements of the acquisition (waveform digitization) of all the sub-detectors have been defined, and the procurement of the electronics is ongoing. The acquisition and computing infrastructure preparation, including the required software, is well advanced.

In order to optimize the beam for the PADME experiment, in terms of pulse length, positron yield, spot and divergence, dedicated LINAC beam-time studies will be performed during 2017.

\section{Conclusions}

Searches for dark photons are well motivated by recently observed phenomena. The PADME experiment will be able to perform such a search exploiting the present LINAC of the DAФNE facility in just one-two year of running and could be the first to directly constrain the region of invisible decays with a sensitivity on the $A^{\prime}$ mass up to $23.7 \mathrm{MeV} / c^{2}$ in a complete model independent way. With the present schedule of the detector construction the data taking could start in 2018.

An upgrade of the BTF positron beam energy will also allow to extend the sensitivity of PADME to higher mass values.

\section{References}

[1] B. Holdom, Phys. Let. B166, 196 (1986).

[2] M. Pospelov, Phys. Rev. D 80, 095002 (2009).

[3] J. Alexander et al., arXiv:1608.08632[hep-ph].

[4] M. Raggi and V. Kozhuharov, Adv. High En. Phys. 2014959802 (2014).

[5] G. Mazzitelli et al., Nucl. Instrum. Meth. A 515, 524 (2003).

[6] M. De Feudis et al., Nuovo Cimento 39 C, 254 (2016). 
[7] I. Valin et al., JINST 7, C01102 (2012).

[8] B. Adeva et al., Nucl. Instrum. Meth. A 289, 35 (1990).

[9] http://hzcphotonics.com.

[10] CAEN Mod. 1742, Technical Information Manual, rev6 06 February 2016.

[11] M. Raggi et al., arXiv:1611.05649[physics.ins-det], submitted to Nucl. Instrum. Meth. A. 